\title{
Correlatos psicosociales del consumo de sustancias psicoactivas en adolescentes españoles
}

\author{
María de la Villa Moral, Dra en Psic, ${ }^{(1)}$ Francisco Javier Rodríguez, Dr en Psic, ${ }^{(2)}$ \\ Anastasio Ovejero, Dr en Psic. ${ }^{(3)}$
}

\author{
Moral MV, Rodríguez FJ, Ovejero A. \\ Correlatos psicosociales del consumo \\ de sustancias psicoactivas en adolescentes españoles. \\ Salud Publica Mex 2010;52:406-4I5.
}

\begin{abstract}
Resumen
Objetivo.Analizar los factores que determinan el consumo juvenil de sustancias psicoactivas evaluando la capacidad predictiva de variables actitudinales, afectivas, relacionales a nivel familiar y grupal, así como académicas, entre otras. Material y métodos. Se ha seleccionado una muestra de 750 adolescentes del Principado de Asturias (España) (moda I5 años, media=14.69) escolarizados en el curso académico 2008-2009. Resultados. Se ha confirmado que los adolescentes que consumen alcohol y otras drogas presentan un perfil diferencial respecto a los no consumidores en factores relativos a la mayor permisividad actitudinal, efecto de modelado paterno y del grupo de iguales, insatisfacción institucional, inestabilidad emocional y manifestación de más conductas disruptivas con problemas de relación y búsquedas de atención. Conclusiones. A partir de nuestro modelo etiológico se concluye que el consumo juvenil de drogas está determinado por la interrelación de variables personales, familiares, escolares y psicosociales.
\end{abstract}

Palabras clave: consumidores de drogas; adolescente; psicología; impacto psicosocial; etiología; apoyo social; España
Moral MV, Rodríguez FJ, Ovejero A.

Psychosocial correlations of the experimentation with psychoactive substances in Spanish teenagers. Salud Publica Mex 2010;52:406-4I5.

\section{Abstract}

Objective. To offer an analysis of the factors that determine the consumption of psychoactive substances by youth and to evaluate the predictive capacity of variables related to attitude, affect, the family and groups, as well as academics, among others. Materials and Methods. A sample of 750 adolescents was selected at the Principado de Asturias (Spain) (mode 15 years of age, mean=14.69) enrolled during the 2008-2009 academic year. Results. It was confirmed that the teenagers who consumed alcohol and other drugs present a differential profile as compared to non-consumers with respect to greater attitudinal permissiveness, effect of paternal modelling and peer groups, institutional dissatisfaction, emotional instability, and manifestation of more disruptive conducts with problems relating to others and attention-seeking. Conclusions. From our ethiological model we conclude that the consumption of drugs by youth is determined by an interrelation of personal, familiar, school and psychosocial variables.

Key words:psychoactive agents; teenagers; psychology; psychosocial impact; etiology; social support, Spain

(I) Área de Psicología Social. Universidad de Oviedo. Oviedo, España

(2) Área de Personalidad, Evaluación y Tratamiento. Universidad de Oviedo. Oviedo, España

(3) Área de Psicología Social. Universidad de Valladolid.Valladolid, España 
$\mathrm{E}_{\mathrm{p}}^{1}$ consumo juvenil de alcohol y otras sustancias psicoactivas sigue representando en el arco mediterráneo, y concretamente en España, un problema sociosanitario de primera magnitud, no sólo por la elevada prevalencia y por los riesgos para la salud física y psíquica de los adolescentes consumidores, sino por las implicaciones psicosociales y comunitarias derivadas de tales usos / abusos. En cambio, los propios consumidores tienden a desarrollar mentalidades del usuario indicativas de una percepción distorsionada de la nocividad de tales prácticas, así como una baja percepción de riesgo asociada. ${ }^{1-5}$ De acuerdo con los resultados de diversos informes institucionales sobre el consumo de alcohol en España publicados por el Plan Sobre Drogas, ${ }^{6-8}$ en la última década se ha producido una reducción significativa de la edad de inicio en el consumo de drogas como el alcohol, que se ha estabilizado alrededor de los 12 años y medio. Según los datos recogidos en las Encuestas Estatales sobre Uso de Drogas en Enseñanzas Secundarias (ESTUDES), dirigidas a estudiantes de 14 a 18 años, realizadas por la Delegación del Gobierno para el Plan Nacional de Drogas (DGPNSD) y cuyos últimos datos publicados corresponden al año 2004, se señala que el alcohol sigue siendo la sustancia más consumida entre los jóvenes de estas edades. En 2004, $82 \%$ de los jóvenes españoles declaraba haberlo consumido alguna vez en la vida, 81\% durante los 12 meses previos a la encuesta y $65.6 \%$ durante los 30 días previos a la misma. Respecto a otras drogas, el cannabis es la droga psicoactiva ilegal más consumida en la Unión Europea, de modo que los adultos jóvenes son los que registran las tasas de consumo más elevadas. En España, la prevalencia mensual de consumo en población escolar es la más alta de Europa. ${ }^{9-10}$ También se informa de que más de la mitad de los usuarios de cannabis se inicia antes de la mayoría de edad. Tales resultados son similares a otros hallados a nivel internacional, entre otros los derivados de la Encuesta Nacional de Adicciones en México, ENA 2008, ${ }^{11}$ y de otros estudios con adolescentes mexicanos. ${ }^{12-18}$

A nivel etiológico, el consumo juvenil de sustancias psicoactivas responde tanto a intentos de satisfacción de motivaciones hedónicas como propiamente a afanes explorativos, prácticas ritualizadas, deseos de experimentación, desajustes socioafectivos, sobreactivaciones varias y a necesidades percibidas de integración en el grupo de iguales y sus estilos de ocio juvenil, propios de una cultura recreativa. ${ }^{19,20}$ En una propuesta comprehensiva de los factores etiológicos implicados en el consumo juvenil de alcohol y de otras drogas ha de aludirse a la interrelación de diversos factores que conforman una suerte de continuum etiológico. ${ }^{21,22}$ En este sentido, esta problemática debería contemplarse como un fenómeno multicausal, de acuerdo con las valoraciones del National Institute on Drug Abuse, de EUA. ${ }^{23-25}$

En la literatura sobre el tema se encuentra apoyo a propuestas etiológicas de base psicosocial (actitudes, modelado, identidad, etc.) como determinantes del consumo de drogas, posicionamiento defendido en este estudio. Por extensión, la implicación etiológica de variables caracteriológicas que actúan como factores de riesgo/ protección también se considera sumamente pertinente, de modo que los estilos y rasgos de personalidad en los adolescentes también intervienen como predictores, ${ }^{26-28}$ mayormente en aquéllos con desajustes psicoafectivos y sociocomportamentales y crisis en la construcción y reformulación de su identidad psicosocial. ${ }^{4,5}$

Está bien establecida la tendencia a que la búsqueda de sensaciones represente uno de los principales motivadores de consumo, tanto de alcohol como de otras drogas, de modo que la curiosidad por la experimentación y la conducta impulsiva constituyen factores que determinan la iniciación al consumo. ${ }^{29}$ En relación con la influencia del estado anímico, se ha hecho referencia a la intolerancia psicofísica al estrés ${ }^{30} \mathrm{o}$ malestar emocional, potenciado por las propias crisis del estado anímico de la condición de adolescente, de modo que se recurre a las drogas como modalidad de escape de interacciones caracterizadas por un conflicto continuo y como mecanismo de afrontamiento/huida. ${ }^{31,32}$ Tendencias a la agresividad temprana, así como conductas antisociales y predictores tales como hiperactividad, rebeldía, pobre autocontrol, dificultad para relacionarse, alienación y disconformidad con las normas, presentan una mayor asociación con el uso problemático de drogas. ${ }^{33}$

Las crisis de identidad en el adolescente representan un factor crítico en la génesis del consumo, así se ha relacionado la iniciación al consumo con el acto de socialización en el grupo de iguales ${ }^{34,35}$ y resulta ser contingente la pertenencia al grupo con la adopción de sus normas reguladoras. Un sentimiento infravalorado de uno mismo, así como déficits interpersonales varios -tales como escasas y/o inadecuadas habilidades sociales, desequilibrios en las habilidades de asertividad y afrontamiento, entre otras-, pueden favorecer la iniciación al consumo de drogas. ${ }^{36,37}$

Bajo la etiqueta de factores de riesgo/protección propios de escenarios vitales se incluye la acción socializadora de la familia (modelado paterno, cambios en la estructura familiar, actitudes paternas hacia las drogas, estilos educativos paternos, hábitos de consumo, etc. $)^{38-40}$ y del grupo de iguales (presión grupal, hábitos de consumo, actitudes permisivas, etc.) ${ }_{1}^{41}$ así como la implicación de múltiples factores escolares como factores de riesgo 
(insatisfacción institucional, movilidad escolar, fracaso académico, inadaptación al clima escolar, baja motivación académica, etc.). $22,42,43$

En suma, el propósito de esta investigación es ofrecer una aproximación psicosocial a la etiología del consumo juvenil de alcohol y de drogas ilegales, con especial énfasis en la implicación de variables vinculadas a la esfera afectiva y socioemocional, así como propias de la percepción del adolescente de la relación conflictuada en los ámbitos socializadores por excelencia (familia, grupo de iguales y escuela), de acuerdo con la naturaleza multidimensional del objeto de estudio y las siguientes hipótesis de investigación:

a) se prevé que el consumo juvenil de alcohol esté determinado por la acción de factores personales y psicosociales, tales como actitudes hacia el consumo, los efectos del modelado paterno y grupal, problemas en la relación con los padres e insatisfacción institucional, entre otros;

b) en el caso del consumo de drogas ilegales se prevé mayor acción determinante de los conflictos psicoafectivos y emocionales, crisis de identidad, autoconcepto general, autoestima y problemas socio-relacionales, así como de la presión grupal por parte de amigos consumidores de drogas ilegales y de los déficits en habilidades sociales como factores de riesgo; $y$

c) en ambos casos, dada la multideterminación del objeto de estudio, se prevé la interrelación de factores personales y psicosociales descritos, así como familiares (estilo educativo paterno/materno y percepción valorativa de los vínculos paterno-filiales, modelado), interaccionales (relaciones conflictuadas con el grupo de iguales) y académicos (actitudes hacia la escuela e insatisfacción institucional).

\section{Material y métodos}

\section{Participantes}

El estudio se ha realizado en cuatro centros de enseñanza secundaria de la zona central del Principado de Asturias (España), una vez evaluados los informes de la Sociedad Asturiana de Estudios Económicos e Industriales (SADEI) sobre el estudio estadístico de los municipios asturianos. En el proceso de selección de los centros de secundaria que han participado en la investigación se ha empleado un muestreo no aleatorio o de juicio, valorando la experiencia del equipo investigador en la zona seleccionada. Dada la aplicación en el entorno escolar, los sujetos ya estaban agrupados mediante un muestreo estratificado (grupos o clases ya conformados) y se ha empleado la técnica de muestreo sistemático, ya que el universo poblacional es de gran tamaño y ha de extenderse en el tiempo. En cada centro se ha empleado un muestreo por conglomerados y con posterioridad se ha aplicado un diseño de muestreo bietápico con selección aleatoria de los conglomerados (27 grupos escolares de todo el universo posible) y muestreo por cuotas de los sujetos participantes en función de la variable edad. En nuestro caso, se ha enfocado la investigación a un estrato poblacional que abarca, básicamente, desde la primera adolescencia (12-13 años) y la adolescencia media (14-16 años) hasta edades más avanzadas (>16 años), estando situada la moda en 15 años (media=14.59). En concreto, la muestra está integrada por 750 alumnos (388 chicas que representan 51.80\% de la muestra y 362 chicos que conforman el $48.2 \%$ restante). Provienen de hogares de clase media, en los que en $61.1 \%$ ( $n=347)$ de los mismos el padre desarrolla la labor de obrero cualificado, y donde la categoría de prejubilados y jubilados es la segunda en importancia $(18.8 \%, \mathrm{n}=107)$. Por lo que respecta al estatus profesional materno, las características más sobresalientes responden a la siguiente distribución: un porcentaje bastante elevado desempeñan la labor de amas de casa $(70.9 \%, \mathrm{n}=411)$, un porcentaje mucho más reducido $(16,8 \%, \mathrm{n}=97)$ al sector servicios o poseen la categoría de obreras cualificadas $(5.5 \%, \mathrm{n}=32)$ o sin cualificar $(5.8 \%, \mathrm{n}=34)$, entre otras. En relación con el nivel cultural, la categoría de estudios primarios es la más frecuente (61.1 y 65.8\% para los niveles paternos y maternos respectivamente). En relación con las consideraciones éticas exigibles a toda investigación, todos los participantes leyeron una carta de consentimiento informado, se recabó información solamente de quienes aceptaron participar $y$, dado que se trata de menores de edad, los padres o tutores firmaron una carta de asentimiento previo a que el menor proporcionara la información requerida por la encuesta. Se hizo énfasis en el carácter voluntario de la participación, así como en la confidencialidad de la información aportada.

\section{Instrumentos de evaluación y materiales}

De acuerdo con las recomendaciones de la Delegación del Gobierno para el Plan Nacional sobre Drogas (DGPNSD, España) en esta investigación se han empleado diversos indicadores de consumo (experimentación, consumo semanal, consumo de riesgo y dependencia). En este artículo en concreto, dado nuestro interés investigador, se ha empleado para los análisis el indicador relativo al consumo de alcohol y/o de otras drogas en el último año y en el último mes, para determinar la prevalencia de consumo y, posteriormente, los factores explicativos del fenómeno objeto de análisis. 
También se han evaluado las actitudes hacia el consumo medidas mediante el factor Actitud global hacia el consumo de alcohol y otras drogas, obtenido en la estructura factorial a partir de la aplicación del Autoinforme de Consumo de Sustancias Psicoactivas (AICA) ${ }^{44}$ que consta de 30 ítems medidos en una escala Likert con cinco niveles de respuesta (MA a MD) y explora actitudes hacia el consumo de alcohol y otras drogas de acuerdo con una triple dimensionalidad: cognitiva (creencias y expectativas), emocional (sentimientos y valoraciones) y comportamental (disposición para actuar), edad de inicio, historia de consumo de alcohol y drogas ilegales y modelos de consumidores (familia y grupo de iguales). Se confirma la existencia de una adecuada validez interna y fiabilidad y se hallan puntuaciones elevadas (>.92) en el indicador de Cronbach, lo que es prueba de una buena consistencia interna, con el valor del coeficiente Alpha de .9314.

Se ha procedido al estudio del autoconcepto concebido como un constructo pluridimensional y jerárquico evaluado mediante el Self Description Questionnaire (SDQ-II), ${ }^{45}$ adaptado a población española e integrado por las siguientes dimensiones: general (DGEN), apariencia física (DAFI), habilidad física (DHFI), matemática (DMAT), verbal (DVER), resto de asignaturas (DRAS), honestidad (DHON), estabilidad emocional (DEEM), relación con los padres (DRCP) y relación con los iguales (DRIGG). Respecto a los resultados hallados sobre la fiabilidad total del instrumento SDQ-II se ha obtenido una adecuada consistencia interna para una muestra válida de 652 adolescentes y 150 reactivos medidos en una escala Likert con cinco niveles de respuesta (MA a MD) (Alfa de Cronbach=.9359).

Para la medida de los trastornos de índole afectivo y emocional se ha utilizado el instrumento Youth Self Report (YSR) creado por Achenbach ${ }^{46}$ para el nivel de edad de 11 a 18 años y adaptado a población española. Conforme a la revisión de análisis previos de la estructura factorial del instrumento, suelen obtenerse siete factores subyacentes que integran el mismo: depresión / ansiedad (F1DA); conducta disruptiva (F2CD); conducta agresiva (F3CA); quejas somáticas (F4QS); problemas de pensamiento (F5PP); problemas de relación (F6PR) y, finalmente, búsqueda de atención (F7BA). El porcentaje total de varianza explicado es $43.8 \%$, correspondiendo al factor 1 la mayor capacidad explicativa (21.6\%) y significativamente menor en los siguientes. El valor del coeficiente Alfa para una muestra de 587 casos válidos y 112 ítems evaluados en formato verdadero / falso es bastante elevado (.9440).

Se ha empleado el Cuestionario de Habilidades Sociales ${ }^{47}$ que consta de 20 reactivos medidos mediante escala Likert con cinco niveles de respuesta (MA a MD) que evalúan asertividad, habilidades de comunicación o resistencia a la presión de grupo, entre otras variables. De acuerdo con la estructura factorial, se explora asertividad y control de situaciones (resistencia a la presión e incitación grupal al consumo de alcohol, manifestación de conductas asertivas y de control de la situaciones asociado al liderazgo grupal), autoafirmación (estrategias de afirmación personal) y habilidades sociales comunicacionales (expresión gestual y la conducta atencional, apertura y expresión emocional, tendencia a expresar las opiniones propias, etc.), así como el factor escasas y/o desadaptativas habilidades sociales y expresión del malestar $y$ defensa de derechos que completa la estructura factorial (Alfa $=.805$ ).

Asimismo, se han evaluado variables académicas (actitudes ante la enseñanza, nivel de aspiración educativa y satisfacción institucional) mediante una escala validada construida ad hoc ${ }^{48}$ que evalúa las percepciones escolares el alumnado y las actitudes hacia la institución académica y está integrada por veinte ítems evaluados mediante escala Likert (MA a MD) adscritos a cuatro factores: satisfacción institucional (valoración de la dedicación del profesorado o su competencia pedagógica y científica, relaciones profesor-alumno, etc.), utilidad práctica de la enseñanza (percepciones valorativas de su validez para garantizar/posibilitar la adquisición de conocimiento, el ingreso en la universidad y la formación integral como persona), Funciones de la educación e inserción sociolaboral (funciones básicas de la educación) y desvalorización (evalúa el rechazo a la institución y crítica a sus funciones y validez práctica). Estos factores explican 63.1 por ciento de la varianza total, correspondiendo al factor 1 el mayor peso explicativo $(37.3 \%)$ y en menor porcentaje a los restantes (13.2, 7.2 y $5.4 \%$, respectivamente), donde resulta elevado (.8805) el valor del coeficiente Alfa de Cronbach.

\section{Procedimiento}

La investigación fue aprobada por la Comisión Ética del Departamento de Psicología de la Universidad de Oviedo (junio de 2008). Se obtuvo el consentimiento informado de los sujetos participantes en el estudio y, de acuerdo con la legislación vigente en España, al ser menores de edad, se obtuvo el consentimiento familiar informado a través de las Asociaciones de Padres de Alumnos (APA) y de los correspondientes Departamentos de Orientación de los Institutos de Enseñanza Secundaria (IES) en los que se llevó a cabo la investigación, como responsables del consentimiento. La aplicación grupal e individualizada de los cuestionarios fue llevada a cabo por investigadores entrenados al efecto. Dado el carácter longitudinal de la investigación se 
asignó un código numérico que únicamente conocía el / la alumno/a correspondiente y el equipo investigador. Se ha respetado escrupulosamente el anonimato y la confidencialidad de los datos y opiniones aportados por el alumnado. En virtud de los acuerdos alcanzados con los departamentos de orientación de cada centro y los tutores correspondientes se aplicaron los instrumentos de evaluación descritos durante un periodo temporal de varias semanas en horario coincidente con actividades de tutorías o libres. Los datos fueron recogidos de forma anónima, aunque se procedió a la asignación de un código numérico (el número correspondiente a cada alumno en clase) de cara a las posteriores labores de intervención psicosocial y sociocomunitaria emprendidas. El procesamiento y análisis estadístico de los datos (análisis factoriales y función discriminante) se ha llevado a cabo mediante el paquete estadístico SPSS versión 15.0.

\section{Resultados}

Como paso previo a la presentación de los resultados obtenidos relativos a los factores determinantes del consumo de alcohol y de otras sustancias psicoactivas se aportan los resultados sobre prevalencia de consumo. Se ha hallado que $63.7 \%$ de los adolescentes encuestados consumen alcohol y 18\% drogas ilegales (cuadro I), evaluado mediante el indicador consumo en el último mes.

De acuerdo con los objetivos planteados se han empleado técnicas de regresión logística con el fin de clasificar a uno y otro grupo de pertenencia predicho a partir de las variables explicitadas, configurando los correspondientes grupos según los cluster de consumo o no consumo. De acuerdo con los resultados hallados, se confirma la implicación de las variables descritas en la predicción y clasificación de los casos presentados, si bien dada la multidimensionalidad del fenómeno objeto de análisis otras variables no investigadas podrían contribuir a clasificarlos de forma adecuada. En su conjunto, en ambos análisis se ha confirmado la adecuación de los valores pronosticados con los observados en las variables de agrupación (consumo de alcohol, por un lado y consumo de drogas ilegales, por otro) a partir de las variables predictoras.

En concreto, en el análisis de la variable de agrupación consumo de alcohol recogido en el cuadro II nuestros hallazgos sugieren que el riesgo de consumo en el grupo de no riesgo pronosticado es de .016, mientras que en el grupo de riesgo en función de las variables predictoras el momio es de 0.91 , siendo la razón de momios (RM) de 5687 con IC (95\%) como cuantificación de la importancia

Cuadro I

TABLA DE CONTINGENCIA GÉNERO, EDAD, CONSUMO JUVENIL DE ALCOHOL Y CONSUMO DE DROGAS ILEGALES. Principado de Asturias, España, 2008

\begin{tabular}{|c|c|c|c|c|c|c|c|c|c|}
\hline \multirow[t]{2}{*}{ Alcohol /drogas ilegales } & & & \multicolumn{6}{|c|}{ Edad } & \multirow[t]{2}{*}{ Total } \\
\hline & & & 12 & 13 & 14 & 15 & 16 & 17 & \\
\hline \multirow[t]{3}{*}{ No consumo de alcohol } & Sexo & Hombre & 18 & 21 & 25 & 22 & 9 & 2 & 97 \\
\hline & & Mujer & 16 & 24 & 31 & 31 & 5 & 4 & 111 \\
\hline & Total & & 34 & 45 & 56 & 53 & 14 & 6 & 208 \\
\hline \multirow[t]{3}{*}{ Consumo de alcohol } & Sexo & Hombre & 13 & 10 & 33 & 66 & 37 & 24 & 183 \\
\hline & & Mujer & 6 & 12 & 48 & 55 & 47 & 18 & 186 \\
\hline & Total & & 19 & 22 & 81 & 121 & 84 & 42 & 369 \\
\hline \multirow[t]{3}{*}{ No consumo de drogas ilegales } & Sexo & Hombre & 30 & 28 & 49 & 71 & 35 & 21 & 234 \\
\hline & & Mujer & 22 & 31 & 66 & 73 & 35 & 15 & 242 \\
\hline & Total & & 52 & 59 & 115 & 144 & 70 & 36 & 476 \\
\hline \multirow[t]{3}{*}{ Consumo de drogas ilegales } & Sexo & Hombre & I & 3 & 9 & 17 & II & 5 & 46 \\
\hline & & Mujer & 0 & 5 & 14 & 13 & 17 & 7 & 56 \\
\hline & Total & & I & 8 & 23 & 30 & 28 & 12 & 102 \\
\hline
\end{tabular}


de la asociación. Se ha confirmado la elevada correspondencia entre los casos observados y pronosticados, de modo que el grupo predicho 2 (consumidores de alcohol) se corresponde con el observado en $90.1 \%$ de los casos, estando correctamente clasificado $80.9 \%$ de los adolescentes que no consumen alcohol en virtud de las covariables seleccionadas.

A partir del análisis de los valores medios obtenidos para cada una de las variables o factores incluidos en los análisis de regresión logística, se ha confirmado que existen diferencias en las puntuaciones medias en los factores descritos, de modo que los valores medios superiores corresponden al segundo grupo pronosticado, en el que se adscribían los adolescentes consumidores de alcohol y de otras drogas, según el análisis. Se constata que los factores que más explican la variable criterio consumo de alcohol son los correspondientes a las actitudes globales ante las drogas, el propio consumo autoinformado de derivados canábicos, la manifestación de indicios de conducta desviada, la insatisfacción institucional hacia el ámbito académico y la influencia de la socialización y el modelado paterno y del grupo de iguales evaluado mediante los correspondientes consumos paternos de alcohol, cannabis y otras drogas ilegales, así como por parte del grupo de iguales.

Asimismo, en la determinación de los mayores riesgos asociados en los grupos de consumo pronosticados se comprueba la existencia de diferencias significativas en indicadores de depresión/ ansiedad en adolescentes consumidores de alcohol respecto a no usuarios, e implicaciones con mayor probabilidad en conductas de búsqueda de atención, tales como las relativas al ausentismo escolar y la desobediencia, o como los ataques físicos y amenazas y en problemas de relación, donde se incluyen conductas de reserva y desconfianza manifiesta hacia los otros y conflictos en los vínculos y en el trato interpersonal. Respecto a las variables sociodemográficas seleccionadas como predictoras no se ha confirmado la existencia de diferencias significativas atribuibles al género $y$, en cambio, se han demostrado las relativas al nivel de estudios en la dirección predicha, con mayor riesgo de consumo en niveles superiores de enseñanza (cuadros II y III).

En el caso concreto del consumo de drogas ilegales, el grupo mejor clasificado es el de adolescentes no consumidores, en el que se ha hallado una correspondencia entre el grupo observado y el pronósticado de $96.6 \%$. Por su parte, $58.2 \%$ de los adolescentes consumidores de drogas ilegales están correctamente clasificados. En virtud de las variables predictoras seleccionadas, el porcentaje global de correspondencia es de $89.1 \%$ (cuadro IV).

En la determinación del objeto de análisis se ha comprobado el valor predictivo como factor de riesgo del indicador relativo a las actitudes permisivas hacia el consumo, así como el consumo autoinformado de alcohol como criterio que discrimina entre los consumidores y no consumidores de drogas ilegales. Ello es aplicable a las diferencias obtenidas en los indicadores expuestos en el consumo familiar de alcohol, cannabis y drogas ilegales, así como en el consumo de cannabis y drogas ilegales por parte de los amigos. A partir del análisis de los efectos evaluados se ha hallado la razón de momios de factores como el de depresión/ansiedad, con cuya etiqueta nominativa se evalúan baja autoestima, sentimientos de inferioridad y soledad, confusión emocional, manifestaciones somáticas de ansiedad, así como la expresión de sentimientos vinculados a la autopercep-

Cuadro II

Regresión logística para CONSUMO JUVENIL de ALCOHOL. TABLA DE CLASIFICACIÓn Y estadísticos. Principado de Asturias, España, 2008

\begin{tabular}{|c|c|c|c|c|c|c|c|}
\hline \multicolumn{3}{|c|}{ Observado } & \multicolumn{5}{|c|}{ Pronosticado } \\
\hline & & & \multicolumn{2}{|c|}{ Alcohol } & & \multirow{2}{*}{\multicolumn{2}{|c|}{$\begin{array}{c}\text { Porcentaje correcto } \\
\end{array}$}} \\
\hline & & & I & 2 & & & \\
\hline \multirow[t]{4}{*}{ Paso I } & Alcohol & I No consumo & 155 & 40 & & & \\
\hline & & 2 Consumo & 33 & 301 & & & \\
\hline & Porcentaje global & & & & & & \\
\hline & & B & E.T. & Wald & $\mathrm{Gl}$ & Sig. & $\operatorname{Exp}(B)$ \\
\hline Paso 0 & Constante & .538 & .090 & 35.655 & I & .000 & 1.713 \\
\hline \multirow[t]{2}{*}{ Pasol } & & & \multicolumn{2}{|c|}{-2 log de la verosimilitud } & $\mathrm{R}^{2}$ de Cox & \multirow{2}{*}{\multicolumn{2}{|c|}{$\begin{array}{c}\mathrm{R}^{2} \text { de Nagelkerke } \\
.705\end{array}$}} \\
\hline & & & \multicolumn{2}{|c|}{$312.649^{*}$} & .516 & & \\
\hline *Valor & .500 & & & & & & \\
\hline
\end{tabular}


Cuadro III

Variables en el modelo de Regresión logística para consumo juVENIL de ALCOHOl (G.L.=I).

Principado de Asturias, España, 2008

\begin{tabular}{|c|c|c|c|c|c|c|c|c|}
\hline \multirow[b]{2}{*}{ Paso I (a) } & \multirow[b]{2}{*}{ Actitudes globales hacia las drogas } & \multirow{2}{*}{$\begin{array}{c}B \\
1.465\end{array}$} & \multirow{2}{*}{$\begin{array}{l}\text { E.T. } \\
.329\end{array}$} & \multirow{2}{*}{$\begin{array}{l}\text { Wald } \\
\text { |2.55| }\end{array}$} & \multirow{2}{*}{$\begin{array}{c}\text { Sig. } \\
.000^{*}\end{array}$} & \multirow{2}{*}{$\begin{array}{l}\operatorname{Exp}(B) . \\
3.205\end{array}$} & \multicolumn{2}{|c|}{$\begin{array}{c}\text { IC } 95 \% \\
\text { Inferior Superior }\end{array}$} \\
\hline & & & & & & & 1.683 & 5.106 \\
\hline & Consumo de cannabis & 2.174 & .908 & 4.035 & $.025^{\ddagger}$ & 8.790 & 1.054 & 13.307 \\
\hline & Consumo familiar de alcohol & 1.184 & .167 & 2.215 & $.042^{\ddagger}$ & 1.202 & .067 & 1.667 \\
\hline & Consumo familiar de cannabis & 1.068 & .468 & 1.005 & $.059 \$$ & .966 & .386 & 2.418 \\
\hline & Consumo familiar de otras drogas ilegales & 1.070 & .995 & 1.040 & $.068^{\S}$ & 1.012 & .489 & 2.046 \\
\hline & Consumo amigos de alcohol & 1.023 & .277 & 2.011 & $.049 \ddagger$ & 1.024 & .656 & 1.597 \\
\hline & Consumo amigos de cannabis & 1.025 & .229 & 1.127 & $.044^{\ddagger}$ & 1.075 & .623 & 1.528 \\
\hline & Consumo amigos de otras drogas ilegales & 1.390 & .838 & 2.175 & $.03 \mathrm{I}^{\ddagger}$ & 2.479 & .480 & 4.804 \\
\hline & Autoconcepto general & .167 & .229 & .532 & .466 & I.182 & .754 & 1.854 \\
\hline & Inestabilidad emocional & .037 & .183 & .684 & .568 & 1.038 & .725 & 1.486 \\
\hline & Relación conflictiva con los padres & 1.918 & .154 & 2.414 & $.029 \ddagger$ & 1.018 & .754 & 1.376 \\
\hline & Relación conflictiva con los iguales & 1.365 & .206 & 3.148 & $.023^{\ddagger}$ & 1.994 & .464 & 2.103 \\
\hline & Depresión/ansiedad & 1.065 & .059 & 1.203 & $.041^{\S}$ & .937 & .834 & 1.052 \\
\hline & Conducta desviada & 5.847 & .922 & 48.099 & $.000 *$ & 2.332 & 1.836 & 2.962 \\
\hline & Conducta agresiva & .061 & .067 & .976 & .511 & 1.009 & .878 & 1.140 \\
\hline & Problemas de relación & 1.141 & .181 & 1.514 & $.032^{\ddagger}$ & .960 & .773 & 1.193 \\
\hline & Búsqueda de atención & 1.181 & .125 & 1.395 & $.044^{\ddagger}$ & .923 & .737 & 1.155 \\
\hline & Déficits en habilidades sociales & .049 & .239 & .142 & .838 & .952 & .596 & 1.522 \\
\hline & Insatisfacción institucional & 5.595 & .222 & 23.751 & $.000^{\ddagger}$ & .552 & .434 & .701 \\
\hline & Sexo & .425 & .339 & 1.069 & .210 & 1.530 & .787 & 1.975 \\
\hline & Nivel académico & 1.710 & .155 & 20.868 & $.000^{\ddagger}$ & 2.034 & 1.500 & 2.758 \\
\hline $\begin{array}{l}* p<.001 \\
\ddagger p<.05 \\
\S p<.10\end{array}$ & & & & & & & & \\
\hline
\end{tabular}

Cuadro IV

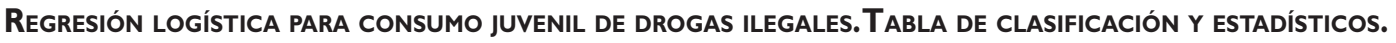
Principado de Asturias, España, 2008

Observado

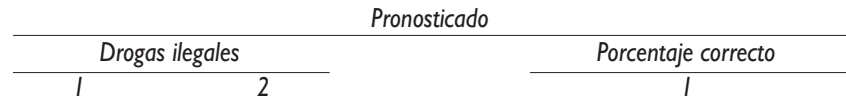

\begin{tabular}{|c|c|c|c|c|c|c|c|}
\hline \multirow[t]{4}{*}{ Paso I } & Drogas ilegales & I No consumo & 422 & 15 & & & \\
\hline & & 2 Consumo & 32 & 60 & & & \\
\hline & Porcentaje global & & & & & & \\
\hline & & B & E.T. & Wald & GI & Sig. & $\operatorname{Exp}(B)$ \\
\hline Paso 0 & Constante & -1.558 & .115 & $184.5 \mid 4$ & I & .000 & .211 \\
\hline
\end{tabular}

Paso I $\begin{array}{ccc}-2 \text { log de la verosimilitud } & \mathrm{R}^{2} \text { de Cox } & \mathrm{R}^{2} \text { de Nagelkerke } \\ 279.442^{*} & .327 & .542\end{array}$

$*$ Valor de corte $=.500$ 
CuadroV

Variables en el modelo de regresión logística para consumo juVENil de dROGas ilegales (G.L.=I). Principado de Asturias, España, 2008

\begin{tabular}{|c|c|c|c|c|c|c|c|c|}
\hline \multirow[b]{2}{*}{ Paso I(a) } & \multirow[b]{2}{*}{ Actitudes globales hacia las drogas } & \multirow{2}{*}{$\begin{array}{r}B \\
2.809 \\
\end{array}$} & \multirow{2}{*}{$\begin{array}{r}\text { E.T. } \\
.308\end{array}$} & \multirow{2}{*}{$\begin{array}{r}\text { Wald } \\
34.402\end{array}$} & \multirow{2}{*}{$\begin{array}{r}\text { Sig. } \\
.000^{*}\end{array}$} & \multirow{2}{*}{$\begin{array}{l}\operatorname{Exp}(b) \\
6.104\end{array}$} & \multicolumn{2}{|c|}{$\begin{array}{c}\text { IC } 95 \% \\
\text { Inferior Superior }\end{array}$} \\
\hline & & & & & & & 3.335 & 9.171 \\
\hline & Consumo de alcohol & 2.884 & .987 & 7.036 & $.008 *$ & 7.883 & 2.123 & 10.612 \\
\hline & Consumo familiar de alcohol & 1.129 & .201 & 2.412 & $.048^{\ddagger}$ & 1.579 & .592 & 1.904 \\
\hline & Consumo familiar de cannabis & 1.204 & .287 & 2.502 & $.045^{\ddagger}$ & 1.226 & .698 & 2.153 \\
\hline & Consumo familiar de otras drogas ilegales & 1.111 & .809 & 1.909 & $.065^{\S}$ & 1.803 & .783 & 4.372 \\
\hline & Consumo amigos de alcohol & .220 & .275 & .640 & .424 & .803 & .468 & 1.376 \\
\hline & Consumo amigos de cannabis & 1.426 & .228 & 4.323 & $.029 \ddagger$ & 2.517 & .969 & 2.973 \\
\hline & Consumo amigos de otras drogas ilegales & 1.302 & .381 & 2.631 & $.04 I^{\ddagger}$ & 2.353 & .642 & 2.853 \\
\hline & Autoconcepto general & 1.063 & .224 & 2.589 & $.046^{\ddagger}$ & 1.039 & .670 & 1.611 \\
\hline & Inestabilidad emocional & 1.043 & .230 & 2.990 & $.037^{\ddagger}$ & .958 & .668 & 1.374 \\
\hline & Relación conflictiva con los padres & 2.030 & .149 & 3.755 & $.033 *$ & 2.750 & .960 & 2.903 \\
\hline & Relación conflictiva con los iguales & 1.008 & .213 & 1.001 & .234 & 1.458 & .664 & 1.532 \\
\hline & Depresión/ansiedad & 1.021 & .153 & 3.155 & $.035^{\ddagger}$ & 2.021 & .920 & 2.733 \\
\hline & Conducta desviada & 1.044 & .713 & 3.369 & $.033^{\ddagger}$ & 2.045 & .906 & 2.206 \\
\hline & Conducta agresiva & -.039 & .063 & .385 & .535 & 1.040 & .919 & 1.176 \\
\hline & Problemas de relación & 1.086 & .207 & $1.89 \mid$ & $\left..05\right|^{\S}$ & .917 & .744 & 1.131 \\
\hline & Búsqueda de atención & 1.005 & .092 & 1.403 & $.079 \$$ & 1.805 & .839 & 2.204 \\
\hline & Déficits en habilidades sociales & 1.159 & .251 & $\mathrm{I} .706$ & $.062^{\S}$ & 1.853 & .952 & 2.394 \\
\hline & Insatisfacción institucional & 3.023 & .410 & 14.304 & $.000 *$ & 6.063 & 2.777 & 7.846 \\
\hline & Sexo & .038 & .332 & .013 & .909 & .963 & .502 & 1.846 \\
\hline & Nivel académico & 1.060 & .173 & 2.120 & $0.48^{\ddagger}$ & 2.062 & 1.075 & 2.490 \\
\hline $\begin{array}{l}* p<.001 \\
\ddagger p<.05 \\
\S p<.10\end{array}$ & & & & & & & & \\
\hline
\end{tabular}

ción de rechazo e incluso de hostigamiento. También se determina la implicación de conductas desviadas en el que se incluyen desobediencias, amenazas o agresiones físicas; a ello se suman mayores desajustes emocionales y problemas de relación que conducen a un mayor riesgo asociado a la búsqueda de atención. Al igual que en el caso anterior, se ha confirmado el riesgo vinculado a los niveles de enseñanza.

Específicamente como variables determinantes del consumo juvenil de drogas ilegales se ha confirmado el valor predictivo de la inestabilidad emocional y de los conflictos internos en el autoconcepto general con sentimientos de desvalorización individual. Los mayores riesgos asociados al empleo de habilidades sociales desadaptativas (escasa asertividad y control de situaciones, pobre autoafirmación) y a la relación conflictiva con los padres (enfrentamiento generacional y rechazo de la disciplina parental) también quedan demostrados. Asimismo, también se ha confirmado un mayor riesgo predictivo de consumo de drogas ilegales en niveles superiores de enseñanza en relación con los más elementales.

\section{Discusión}

El consumo juvenil de sustancias psicoactivas está determinado por múltiples factores pertenecientes a diversos planos de causalidad que se interrelacionan, con cuyo análisis se trata de dilucidar la importancia relativa de cada cual en la explicación de este fenómeno multideterminado. Los resultados hallados en este estudio van en la dirección de los hallazgos de otras investigaciones citadas en el abordaje teórico de la cuestión tanto en España $^{22,26,29}$ como en la realidad latinoamericana ${ }^{12,15,18}$ y en el contexto anglosajón. 28,41

Se ha confirmado que la mayor permisividad actitudinal, las creencias distorsionadas sobre los efectos de las drogas, el modelado paterno y del grupo de iguales 
consumidores de drogas, la escasa resistencia a la incitación grupal, la tendencia permisiva a permanecer en ambientes y con compañías incitadoras al consumo, entre otras variables, pronostican el consumo de alcohol y de otras drogas. En la línea de otras investigaciones ${ }^{14,16,22-24}$ se han obtenido efectos diferenciales sobre los hábitos de consumo de sustancias psicoactivas en función de los indicadores de permisividad actitudinal, modelado paterno y del grupo de iguales, relaciones conflictuadas en el ámbito familiar, insatisfacción institucional, conducta agresiva y de búsqueda de atención, entre otras.

En concreto, en este estudio se ha comprobado la implicación de factores tanto de índole psicoafectivo (oscilaciones en el estado anímico, depresión/ ansiedad, agresividad temprana, síndromes de patología externa e interna, pobre autocontrol, etc.) como psicosociológico (búsqueda de atención, conducta disruptiva, escasas habilidades sociales, etc.) y de identidad. Estos factores interactúan con otros de carácter microsocial asociados a las agencias socializadoras por excelencia, tanto en el ámbito familiar (relación conflictiva con los padres, enfrentamiento generacional y rechazo de la disciplina parental) como en el académico (insatisfacción institucional) que determinan la problemática del consumo juvenil de drogas. De ahí la propuesta de un modelo de evaluación e intervención integrador a modo de continuum etiológico, ${ }^{21,22}$ mediante el que se superen las propias limitaciones de este estudio, tales como las relativas a la determinación de la problemática por variables no sólo personales y psicosociales sino también de índole fisiológica (vulnerabilidades) y macrocontextual (disponibilidades de la sustancia, cultura estereotípica de la droga, etc.) que no han sido incluidas en el análisis y que, de acuerdo con una evaluación comprehensiva del fenómeno, se interrelacionan en la causación final de esta problemática.

Respecto a las posibles limitaciones del estudio, los resultados de este trabajo no establecen relaciones casuales debido a que se trata de un estudio epidemiológico descriptivo con falta de temporalidad. Asimismo, inciden en las limitaciones propias del carácter transversal del estudio, así como en las derivadas de la selección muestral no aleatoria, los inconvenientes del autoinforme (subestimación del fenómeno), a pesar de estar documentada la validez de los cuestionarios autoaplicados y los derivados del empleo de medidas autoinformadas (sesgo de deseabilidad social), los cuales se intentaron contrarrestar mediante la garantía del anonimato y la confidencialidad de los datos.

Como conclusiones generales y futuras líneas de actuación se considera que cuanto antes construya el adolescente su identidad personal y psicosocial podrá enfrentarse más eficazmente a las presiones que pue- den inducirle al consumo de sustancias psicoactivas. ${ }^{1,4}$ Dotarles de habilidades para la vida repercutirá en su manejo de habilidades cognitivas y relacionales, en su autorregulación exitosa, así como en la posesión y empleo de estrategias que les permitan enfrentarse adaptativamente a diversos estresores y a conflictos emocionales intra e interpersonales, lo cual fortalecerá su capacidad de resiliencia. ${ }^{27,49,50}$

También se insiste en la necesidad de promover cambios en los estilos educativos paternos y en las dinámicas comunicativas intrafamiliares, en la dirección de un reforzamiento de las normas como factores protectores de los consumos de drogas. Por extensión, el diseño e implementación de programas de prevención primaria y secundaria en las escuelas y de intervención psicosocial representa un reto sociosanitario, cuyas líneas prospectivas de actuación han de contemplar la multicausalidad de la problemática descrita.

\section{Declaración de conflicto de intereses}

Declaramos no tener conflicto de intereses.

\section{Referencias}

I. Calafat A. El abuso de alcohol de los jóvenes en España.Adicciones 2007;19:217-223.

2. Bach i Bach L. La falta de conciencia del daño alcohólico, firme impedimento para la prevención primaria y secundaria del alcoholismo. Rev Esp Drogodependencias 2000;25: I |4- I I7.

3. Castellana M, Lladó M.Adolescencia y juventud: Prevención y percepción del riesgo al consumo. Rev Esp Drogodependencias 1999;24:II8-130.

4. Moral MV, Ovejero A.Análisis diferencial por niveles de edad de las actitudes hacia el consumo de sustancias psicoactivas en adolescentes españoles. Interam J Psychol 2005;39:325-338.

5. Salvador CM. Expectativas de los jóvenes respecto al consumo de alcohol: La práctica del botellón. Rev Esp Drogodependencias 2009;34:62-73.

6. Delegación del Gobierno para el Plan Nacional sobre Drogas. Encuesta sobre Drogas a la Población Escolar 2003. Madrid: DGPNSD Ministerio del Interior, 2004.

7. Delegación del Gobierno para el Plan Nacional sobre Drogas. Encuesta sobre Drogas a la Población Escolar 2004. Madrid: DGPNSD Ministerio del Interior, 2005.

8. Delegación del Gobierno para el Plan Nacional sobre Drogas. Informe sobre el alcohol en España. Madrid: DGPNSD Ministerio del Interior, 2007. 9. Ministerio de Sanidad y Política Social Gobierno de España. Consumo problemático de cannabis en estudiantes españoles de 14-18 años: validación de escalas. Madrid: DGPNSD, 2009.

10. Delegación del Gobierno para el Plan Nacional sobre Drogas. Cannabis II. Informe $n^{\circ} 4$ de la Comisión Clínica. Madrid: DGPNSD, Ministerio de Sanidad y Política Social Gobierno de España, 2009.

I I. Instituto Nacional de Salud Pública. Encuesta Nacional de Adicciones 2008. México: Instituto Nacional de Salud Pública INSP, Consejo Nacional contra las Adicciones CONADIC, Instituto Nacional de Psiquiatría Ramón de la Fuente, Fundación González Río Arronte IAP, 2009. 
12.Arellanez-Hernández JL, Díaz-Negrete DB, Wagner-Echegaray F, PérezIslas V. Factores psicosociales asociados con el abuso y la dependencia de drogas entre adolescentes: análisis bivariados de un estudio de casos y controles. Salud Ment 2003;27:54-64.

13. EMA. Examen Médico Automatizado para Estudiantes. Generación 2005. Dirección General de Servicios de México. Secretaría Administrativa UNAM, 2005

14. Fleiz C, Borges G, Rojas E, Benjet C, Medina-Mora ME. Uso de alcohol, tabaco y drogas en población mexicana, un estudio de cohortes. Salud Ment 2007;30:63-73.

15. Herrera-Vázquez M et al. Inicio en el consumo de alcohol y tabaco y transición a otras drogas en estudiantes de Morelos, México. Salud Publica Mex 2004;46:132-I40.

16. Medina-Mora ME, Cravioto P,Villatoro J, Fleiz C, Galván-Castillo F, Tapia-Conyer R. Consumo de drogas entre adolescentes: resultados de la Encuesta Nacional de Adicciones. Rev Socidrogalcohol 1998. Salud Publica Mex 2003;45:516-525.

17. Sánchez-Zamorano LM, Llerenas MA,Anaya-Ocampo R, LazcanoPonce E. Prevalencia del uso de drogas ilegales en función del consumo de tabaco en una muestra de estudiantes en México. Salud Publica Mex 2007;49:182-193.

18. Urquieta JE, Hernández-Ávila M, Hernández B. El consumo de tabaco y alcohol en jóvenes de zonas urbanas marginadas de México: un análisis de decisiones relacionadas. Salud Publica Mex 2006;48 supll:30-40. 19. Graña JL, Muñoz MJ. Factores psicológicos de riesgo y de protección para el consumo de drogas en adolescentes. Psicología Conductual 2000;8:249-269.

20. Martínez JM, Robles L.Variables de protección ante el consumo de alcohol y tabaco en adolescentes. Psicothema 200I;13:222-228. 21. Moral MV, Rodríguez F], Sirvent C. Motivadores de consumo de alcohol en adolescentes:Análisis de las diferencias intergénero y propuesta de un continuum etiológico. Adicciones 2005; 17:105-120. 22. Moral MV, Rodríguez FJ, Sirvent C. Factores relacionados con las actitudes juveniles hacia el consumo de alcohol y otras sustancias psicoactivas. Psicothema 2006; 18:52-58.

23. National Institute on Drug Abuse. Preventing drug abuse among children and adolescents. A research-based guide. NIH Publication $97-$ 4212. Rockville MD: National Institutes of Health, 1997.

24. National Institute on Drug Abuse. Substance abuse and mental health services administration. 2005. Results from the 2004 National Survey on Drug Use and Health: National Findings (Office of Applied Studies, NSDUH Series H-28, DHHS Publication No. SMA 05-4062). Rockville, MD: SAMHSA. NIDA Notes, 2006a;20(6).

25. National Institute on Drug Abuse. Studies identify factors surrounding rise in abuse of prescription drugs by college students. NIDA Notes, 2006b;20(4)

26. Espada JP, Méndez X, Griffin K, Botvin GJ.Adolescencia: consumo de alcohol y otras drogas. Pap Psicol 2003;84:9-17.

27. Moral MV. Personalidad, resiliencia y otros factores psicosociales asociados al consumo de sustancias psicoactivas en la adolescencia: propuesta etiológica. Monografía: Personalidad y consumo de drogas. Rev Esp Drogodependencias 2007a;32:250-29l.

28. Ellickson PL, Tucker JS, Klein DJ, McGuigan KA. Factores prospectivos de riesgo para problemas relacionados con el alcohol en la etapa final de la adolescencia. Journal Studies Alcohol 200I;62:773-782.

29. Martínez-Lorca M,Alonso-Sanz C. Búsqueda de sensaciones, autoconcepto, asertividad y consumo de drogas. ¿Existe relación? Adicciones 2003; 15:145-157.

30. Nadal R. La búsqueda de sensaciones y su relación con la vulnerabilidad a la adicción y al estrés. Adicciones 2008:20:59-72.
31. Braverman M. Research on resilience and its implications for tobacco prevention. Nicotine \& Tobacco Research 200I;I (supl. I): 67-72.

32. Wills TA. Stress and coping in early adolescence: relationships to substance use in urban school samples. Health Psychology 1986;5:503-529. 33. Chan EL, Rodríguez FJ, Moral MV. La conducta delictiva del menor en el estado de Jalisco (México). Análisis diferencial de factores psicosociales asociados, como el consumo de drogas. Monografía Personalidad y consumo de drogas. Rev Esp Drogodependencias 2007;32(3):425-440. 34. Chassing L, Presson CC, Sherman SL, Margolis S. The social image on smokeless tobacco use in three different types of teenagers. Addictive Behaviors 1998;13:107-1 I2.

35. González-Calleja F, García-Señorán MM, González G. Consumo de drogas en la adolescencia. Psicothema 1996;8(2):257-267.

36. López-Torrecillas F, Peralta I, Muñoz-Rivas MJ, Godoy JF.Autocontrol y consumo de drogas. Adicciones 2006;15(2):127-136.

37. Esnaola I.Autoconcepto y consumo de drogas legales en la adolescencia. Rev Esp Drogodependencias 2006; 1:57-66.

38. Musitu G, Jiménez T, Murgui S. Funcionamiento familiar, autoestima y consumo de sustancias en adolescentes: un modelo de mediación. Salud Publica Mex 2007;49:3-10.

39. Nuño-Gutiérrez BL, González-Foreza C. La representación social que orienta las decisiones paternas al afrontar el consumo de drogas de sus hijos. Salud Publica Mex 2004; 46:123-I3I.

40. Tarter RE, Schultz K, Kirisci L, Dunn M. ¿Aumenta el riesgo de abuso de drogas de los hijos varones la convivencia con un padre que abusa de drogas? Impacto sobre los factores de vulnerabilidad del individuo, la familia, la escuela y los compañeros. J of Child \& Adolescent Substance Abuse 200I; 10:59-70.

4I. lannotti RD, Bush PJ, Weinfurt KP. Perception of friends' use of alcohol, cigarettes, and marihuana among urban school children:A longitudinal analysis. Adictive Behavior 1996;5:615-632.

42. López-Frías M, Fernández MF, Planells E, Miranda MT, Mataix J, Llopis J. Consumo de alcohol y rendimiento escolar en una población de estudiantes españoles de secundaria.J Studies on Alcohol 200I;62:74I-744. 43. Torregrosa MS, Inglés CJ, Espada JP, Delgado B, García-Fernández JM, Redondo J. Relaciones entre consumo de alcohol, motivación académica, autoconcepto y rendimiento escolar.Adicciones, XXXIV Jornadas Nacionales de Socidrogalcohol 2007:266-267.

44. Santacreu J, Froján MX. Evaluación del consumo de drogas. En: Fernández-Ballesteros, R. Evaluación conductual hoy. Un enfoque para el cambio en psicología clínica y de la salud. Madrid: Pirámide, 1994:57|-612. 45. Marsh HW.The Self Description Questionnaire (SDQ) II.A Theoretical and empirical basis for measurement of multiple dimensions of adolescents self-concept:An interim test manual and a research monograph. Macarthur, New South Wales, Australia: University of Western Sydney, Faculty of Education, 1992.

46. Achenbach T. Manual for the Youth Self-Report \& 1991 Profile. Burlington: University of Vermont, Department of Psychiatry, 1991. 47. Macià FX, Méndez, J, Olivares D. Intervención psicológica. Programas aplicados de tratamiento. Madrid: Pirámide, 1993.

48. Ovejero A, García Al, Fernández JA. Correlatos psicosociales del fracaso escolar en estudiantes de Bachillerato y Formación Profesional. Psicothema 1996;6:245-258.

49. Becoña E. Resiliencia y consumo de drogas: una revisión. Adicciones 2007;19:89-101.

50. Fergus S, Zimmerman MA.Adolescent resilience:A framework for understanding healthy development in the face of risk. Annu Rev Public Health 2000;26:399-419. 